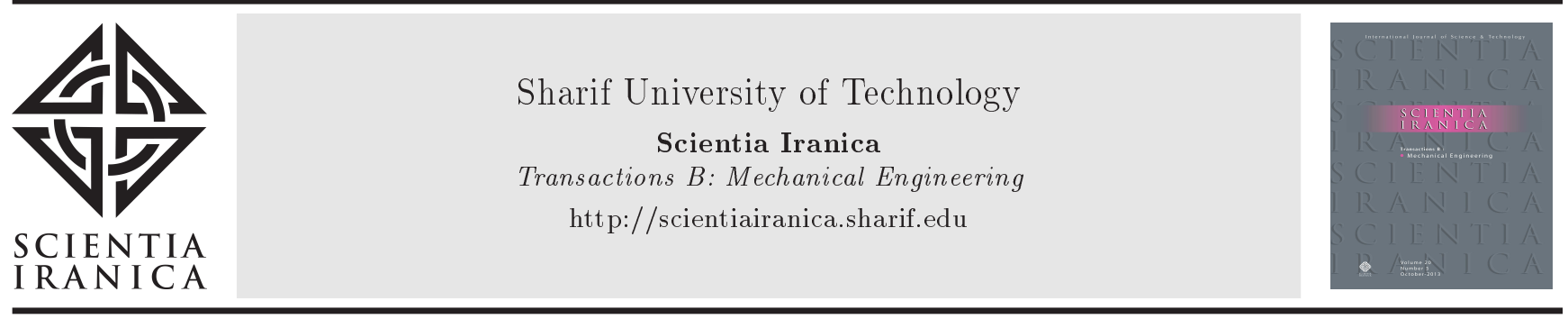

\title{
Application of hyperelastic models in mechanical properties prediction of mouse oocyte and embryo cells at large deformations
}

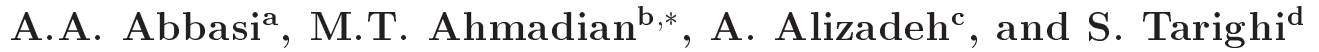 \\ a. School of Mechanical Engineering, Sharif University of Technology, Tehran, P. O. Box 11155-9161, Iran. \\ b. Center of Excellence in Design, Robotics, and Automation (CEDRA), School of Mechanical Engineering, Sharif University of \\ Technology, Tehran, P.O. Box 11155-9161, Iran. \\ c. Institute for Biotechnology \& Environment (IBE), Sharif University of Technology, Tehran, P.O. Box 11155-9161, Iran. \\ d. School of Management and Economics, Sharif University of Technology, Tehran, P.O. Box 11155-9161, Iran.
}

Received 25 January 2016; received in revised form 18 October 2016; accepted 17 April 2017

\author{
KEYWORDS \\ Biological cell; \\ Inverse finite element \\ method; \\ Hyperelastic material; \\ Levenberg-Marquardt \\ optimization \\ algorithm; \\ Large deformation.
}

\begin{abstract}
Biological cell studies have many applications in biology, cell manipulation, and diagnosis of diseases such as cancer and malaria. In this study, Inverse Finite Element Method (IFEM) combined with Levenberg-Marquardt optimization algorithm has been used to extract and characterize material properties of mouse oocyte and embryo cells at large deformations. Then, the simulation results have been validated using data from experimental works. In this study, it is assumed that cell material is hyperelastic, isotropic, homogenous, and axisymmetric. For inverse analysis, FEM model of cell injection experiment implemented in Abaqus software has been coupled with Levenberg-Marquardt optimization algorithm written in Matlab; through this coupling, the optimum hyperelastic coefficients, which give the best match between experimental and simulated forces, are extracted. Results show that among different hyperelastic material models, Ogden material is suitable for characterization of mouse oocyte cell and Mooney-Rivlin or polynomial is suitable for characterization of mouse embryo cell. Moreover, the evaluated Poisson ratio of the cell is obtained to be equal to 0.5 , which indicates that the structural materials of mouse oocyte and embryo are compressible.
\end{abstract}

(C) 2018 Sharif University of Technology. All rights reserved.

\section{Introduction}

Since the structural and functional integrity of general tissues could be determined by their constructive individual cells, understanding and investigating of biological cells behavior are very important [1]. Biological cells, during their life, experience different

*. Corresponding author. Tel.: +982166165503

E-mail addresses: ali.eng.edu@gmail.com (A.A. Abbasi)

ahmadian@sharif.ir (M.T. Ahmadian)

aalizadeh@sharif.edu (A. Alizadeh)

s_tarighi@pgre.iust.ac.ir (S.Tarighi)

doi: $10.24200 /$ sci. 2017.4321 physiological processes such as differentiation, growth, and migration. Therefore, a range of different experimental techniques regarding their environmental conditions have already been proposed for better analyzing and investigation of the complex relationship between a biological cell and its environment, e.g., cell injection $[2,3]$, in which a single cell is injected by a micro or nano needle; shear flow [4], in which fluid shear stress is applied to investigate cell adhesion; cell compression by micro plate [5], in which two micro plates are used to compress a single cell (this technique has been used to measure the mechanical properties of the cell); micropipette aspiration [6] that uses a micropipette to aspirate a single cell by a suction 


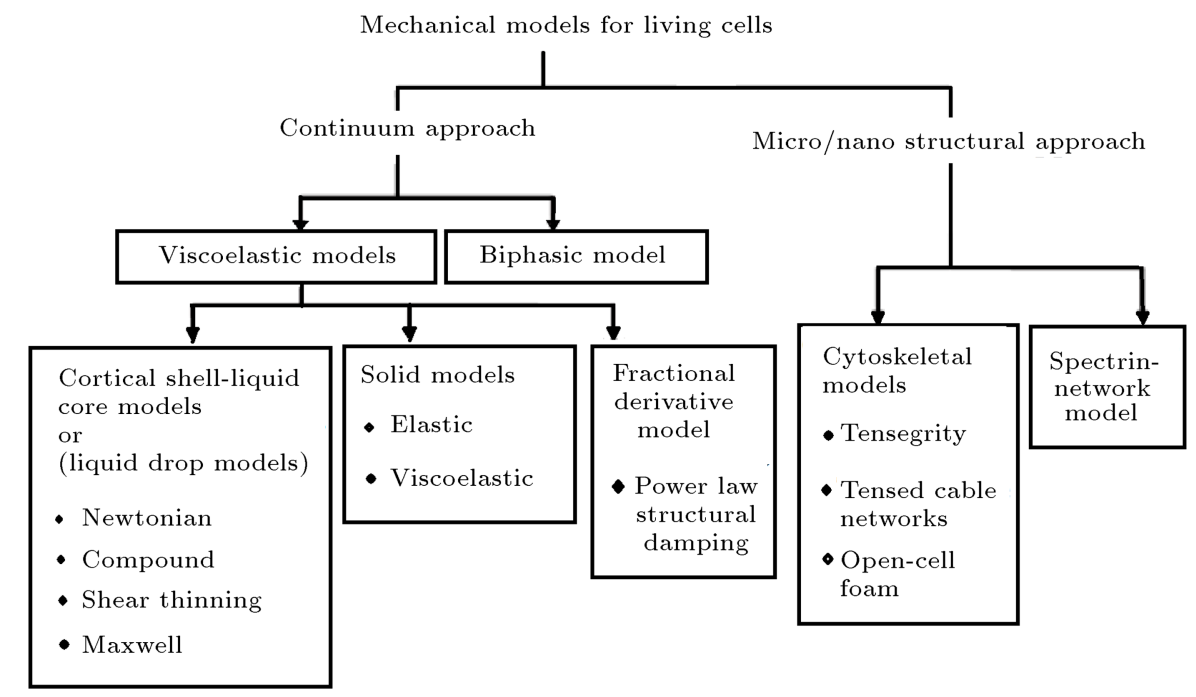

Figure 1. Classification of different mechanical models for living cells [1].

pressure; AFM indentation [7], which is similar to cell injection technique; optical tweezers [8], also known as laser traps, in which a single cell is stretched by optical forces or a laser beam; microarrays for investigation of local cellular environment [9], which can also be used to measure cellular response to environmental stimuli; and Magnetic Twisting Cytometry (MTC) [10,11] and oscillatory magnetic twisting cytometry [1], which are two recent techniques similar to optical tweezers.

Based on these widely various experimental techniques, a range of different mechanical models have been developed for detailed characteristic description of the cells. These models can be classified into two main groups: models which use continuum approaches such as Maxwell, Newtonian, and so on (see Figure 1); and models which use micro/nano structural approaches such as open cell foam, tensegrity [12], and so on (see Figure 1).

In addition, there are numerical models which have been used for prediction of force as well as deformation of cells, e.g. Artificial Neural Network (ANN) [13-18], support vector machine method [19,20], adaptive neural fuzzy inference [21-23], and Inverse Finite Element Method (IFEM) [24]; in these techniques, dimple depth is another input variable considered in the modeling; these models are more suitable for online applications.

Among different numerical approaches, Inverse Finite Element Method (IFEM) has been found to be an effective technique to obtain material properties using experimental data [25-28]. IFEM could be used to solve complex problems, physically or geometrically, with more details and accuracy. Namani and Simha [25] used this method to find the behavior of soft tissue layers. In this regard, they used trial and error approach to find hyperelastic parameters, which needed more calculations to give the optimized parameters; moreover, the study did not consider other hyperelastic materials such as Ogden, Arruda-Boyce, etc. Delalleau and his co-workers used inverse analysis combined with extended Kalman filter to determine the mechanical properties of human skin [26]. However, in Kalman filter method, the divergence of the covariance matrix was a big problem. Moreover, this study did not consider different hyperelastic models. Several investigations used inverse finite element analysis, which focused on small deformation assumption $[27,28]$.

In spite of the fact that all investigations mentioned are useful to study behavior of the biological cells in many cases, challenges in this area still exist. For example, studying the mechanical properties of neutrophil cells using different experimental techniques lead to different mechanical properties for the same cells $[1,29,30]$; therefore, developing optimization-based numerical models could help to achieve better characteristic behavior of the cell.

The present study uses inverse finite element analysis combined with Levenberg-Marquardt optimization algorithm to extract material properties of mouse oocyte and embryo cells at large deformations. With respect to the previous work of the authors [24], which used only Neo-Hookean hyperelastic model, in this study, it is tried to take other hyperelastic models into account. Also, it is shown that classic contact mechanic models are not suitable for large deformation characterization. Different hyperelastic material models, e.g. Arruda-Boyce, Mooney-Rivlin, Neo Hooke, Ogden, Polynomial, reduced polynomial, and Van der Waals, are considered to find the one able to give the best fit with the experimental data. The mechanical model of the cell injection experiment is implemented in Abaqus software [31] and optimization algorithm is written in Matlab [32]. Experimental data of mouse oocyte and embryo cells for data validation 
are captured from the work of Sun et al. [33] and Flückiger [34].

\section{Material models}

As experimental data have been obtained in a timeindependent manner, modeling is based on the Hyperelastic scheme rather than the viscoelastic one. Hyperelastic materials are described using strain energy function, which is usually defined based on strain energy stored in the material per unit of volume in the initial form. In this regard, several forms of three-dimensional strain energy potentials have been investigated such as Arruda-Boyce, Mooney-Rivlin, Neo Hooke, Ogden, Polynomial, reduced polynomial, and Van der Waals. Each model used in this investigation will be described briefly in the next sections.

\subsection{Arruda-Boyce model}

The form of the Arruda-Boyce strain energy potentials is as follows [35] :

$$
\begin{aligned}
U= & \mu\left\{\frac{1}{2}\left(\bar{I}_{1}-3\right)+\frac{1}{20 \lambda_{m}^{2}}\left(\bar{I}_{1}^{2}-9\right)+\frac{11}{1050 \lambda_{m}^{4}}\left(\bar{I}_{1}^{3}-27\right)\right. \\
& \left.+\frac{19}{7000 \lambda_{m}^{6}}\left(\bar{I}_{1}^{4}-81\right)+\frac{519}{673750 \lambda_{m}^{8}}\left(\bar{I}_{1}^{5}-243\right)\right\} \\
& +\frac{1}{D}\left(\frac{J_{e l}^{2}-1}{2}-\ln J_{e l}\right),
\end{aligned}
$$

where, $U$ is strain energy per unit of reference volume, $\bar{I}_{1}$ is first deviatoric strain invariant, $J_{e l}$ is elastic volume ratio, $\mu$ and $\lambda_{m}$ are the temperature-dependent material coefficients, and $D$ is a temperaturedependent material parameter, which controls the compressibility of the material. For typical value of $\lambda_{m}=7$, the initial shear modulus $\mu_{0}$ is related to $\mu$ by [31]:

$$
\mu_{0}=1.0125 \mu \text {. }
$$

$\mu$ and $D$ are obtained from the IFE analysis.

In order to determine cell mechanical properties, Young's modulus (E), and Poisson's ratio $(\nu)$, the initial bulk modulus should be calculated using the following relation [31]:

$$
k_{0}=\frac{2}{D}
$$

Then, Poisson's ratio, $\nu,[31]$ and Young's modulus, $E,[36]$ are determined as follows:

$$
\begin{aligned}
& \nu=\frac{\frac{3 k_{0}}{\mu_{0}}-2}{\frac{6 k_{0}}{\mu_{0}}+2}, \\
& E=2 \mu_{0}(1+\nu) .
\end{aligned}
$$

The above relations, i.e. Eqs. (3)-(5), will also be used in other hyperelastic models to calculate mechanical properties of the cell.

\subsection{Mooney-Rivlin model}

The form of the Mooney-Rivlin strain energy potential is as follows $[37,38]$ :

$$
U=C_{10}\left(\bar{I}_{1}-3\right)+C_{01}\left(\bar{I}_{2}-3\right)+\frac{1}{D_{1}}\left(J_{e l}-1\right)^{2},
$$

in which $C_{10}$ and $C_{01}$ are temperature-dependent material parameters, $D_{1}$ is compressibility, $\bar{I}_{1}$ and $\bar{I}_{2}$ are the first and second deviatoric strain invariants, respectively, and $J_{e l}$ is the elastic volume ratio. The initial shear modulus for this model is derived using the equation below [31]:

$$
\mu_{0}=2\left(C_{10}+C_{01}\right) \text {. }
$$

\subsection{Neo-Hookean model}

The Neo-Hookean strain energy potential form is as follows $[39,40]$ :

$$
U=C_{10}\left(\bar{I}_{1}-3\right)+\frac{1}{D_{1}}\left(J_{e l}-1\right)^{2} .
$$

Again, $C_{10}$ and $D_{1}$ are respectively the temperaturedependent material parameters and compressibility and $J_{e l}$ is the elastic volume ratio. The initial shear modulus is as follows [31]:

$$
\mu_{0}=2 C_{10} \text {. }
$$

\subsection{Ogden model}

The following equation shows the form of the Ogden strain energy potential [41]:

$$
U=\sum_{i=1}^{N} \frac{2 \mu_{i}}{\alpha_{i}^{2}}\left(\bar{\lambda}_{1}^{\alpha_{i}}+\bar{\lambda}_{2}^{\alpha_{i}}+\bar{\lambda}_{3}^{\alpha_{i}}-3\right)+\sum_{i=1}^{N} \frac{1}{D_{i}}\left(J^{e l}-1\right)^{2 i},
$$

where, $\bar{\lambda}_{i}$ is the deviatoric principal stretch, $N$ is the order of the energy function, $D_{i}$ is compressibility, and $\mu_{i}$ and $\alpha_{i}$ describe the shear behavior of the material. The initial shear modulus can also be derived as [31]:

$$
\mu_{0}=\sum_{i=1}^{N} \mu_{i}
$$

\subsection{Polynomial model}

Polynomial strain energy model can be defined as [42]:

$$
U=\sum_{i+j=1}^{N} C_{i j}\left(\bar{I}_{1}-3\right)^{i}\left(\bar{I}_{2}-3\right)^{j}+\sum_{i=1}^{N} \frac{1}{D_{i}}\left(J^{e l}-1\right)^{2 i}
$$

where, $C_{i j}$ is temperature-dependent material parameter, which describes the shear behavior of the material; $N$ is the order of the energy function; $D_{i}$, again, introduces compressibility, and for fully incompressible material it is equal to zero; other parameters are the same as those described above. 


\subsection{Reduced polynomial model}

Reduced polynomial strain energy is described as follows [31]:

$$
U=\sum_{i=1}^{N} C_{i 0}\left(\bar{I}_{1}-3\right)^{i}+\sum_{i=1}^{N} \frac{1}{D_{i}}\left(J^{e l}-1\right)^{2 i},
$$

where, $C_{i 0}$ is temperature-dependent material parameter. $\mu_{0}$ can also be found from Eq. (9). Other parameters are the same as those described above.

\subsection{Van der Waals model}

Van der Waals strain energy model, which is also known as the Kilian model, may be written as [43]:

$$
\begin{aligned}
U= & \mu\left\{-\left(\lambda_{m}^{2}-3\right)[\ln (1-\eta)+\eta]-\frac{2}{3} a\left(\frac{\tilde{I}-3}{2}\right)^{\frac{3}{2}}\right\} \\
& +\frac{1}{D}\left(\frac{J_{e l}^{2}-1}{2}-\ln J_{e l}\right),
\end{aligned}
$$

where, $\lambda_{m}$ is the locking stretch, $\mu$ is the initial shear modulus, $a$ is the global interaction parameter, $D$ controls compressibility, and $\tilde{I}$ and $\eta$ could be defined as [31]:

$$
\begin{aligned}
\tilde{I} & =(1-\beta) \bar{I}_{1}+\beta \bar{I}_{2}, \\
\eta & =\sqrt{\frac{\tilde{I}-3}{\lambda_{m}^{2}-3}},
\end{aligned}
$$

where, $\beta$ is the invariant mixture parameter. Initial bulk modulus may be derived from Eq. (3) and the initial shear modulus can be described as [31]:

$$
\mu_{0}=\mu
$$

\section{Experimental setup}

The experimental system includes a cell holding unit, a vacuum unit, capacitive force sensor unit, an imaging unit, and a software unit (Figure 2). The cell holding unit is a standard pipette, which can fix a biologic cell by applying a sucking pressure through the vacuum unit. Capacitive force sensor includes an injection micropipette, the motion of which is controlled by a

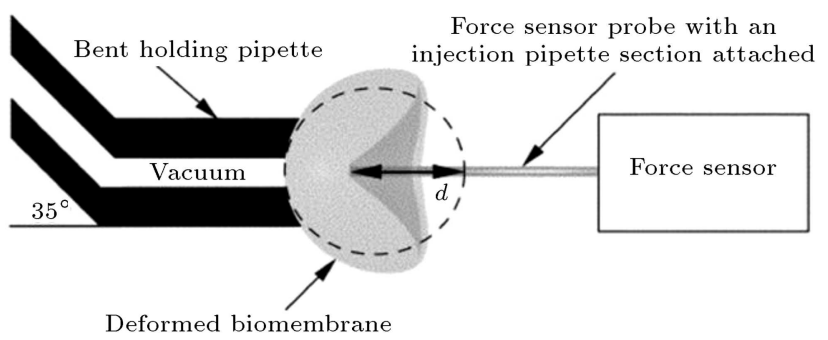

Figure 2. Cell injection system configuration [33].
3-DOF micro robot. A uniaxial compressing load is increasingly applied to the cell until its membrane is ruptured. During this process, the related forces and deformations are recorded by imaging and software units. More details of experimental setup and sample preparations could be found in [33].

\section{Finite element model}

Finite element method of cell injection experiment has been implemented in Abaqus software [31]. The cell has been assumed to be circular with the radius of $25 \mathrm{um}$ before deformation and it has isotropic and homogenous material properties. The simulation is performed under axisymmetric conditions, in which the computation time will be reduced; therefore, the center of the cell is assumed to be fixed in the $x$-direction while it is free to move in the $y$-direction. Because of the axisymmetry, a half of the cell has been used for analysis and, for convenience of expression, a quarter of the cell has been shown (Figure 3 ). The outer boundary of the cell is free to move. The bottom of the cell is in contact with an axisymmetric rigid substrate, which has been restricted in the directions of $x$ and $y$ axes.

The mesh has been refined in the neighborhood of the contact regions, where the largest deformations are expected to happen. The element size in the contact and non-contact areas has been carefully chosen to get a proper accuracy of the computed force. The mesh density has also been carefully chosen and tested to ensure that the solutions converge and acceptable results will be acquired. Dimension of the finite element meshing in the contact area is much smaller than that in other parts of the cell. Information of nodes and elements for the implemented model in Abaqus has been provided in Figure 3 and Table 1.

Initial indenter radius is $3 \mathrm{um}$. Since indenter is much stiffer than the cell, it is approximated as a rigid body. Therefore, in Abaqus, it is defined as analytical rigid body, which is used to model very stiff parts. The indenter geometry and dimension have been illustrated in Figure 4.

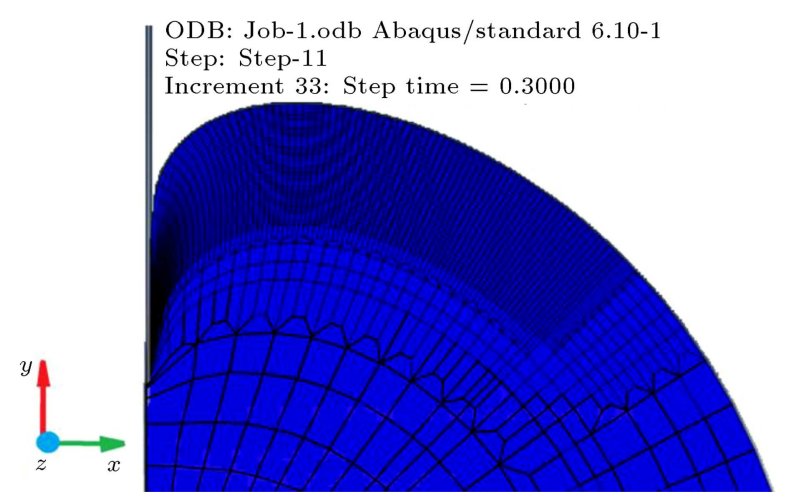

Figure 3. Stress distribution obtained from FE analysis. 
Table 1. Information of nodes and elements of finite element model.

\begin{tabular}{ccc}
\hline $\begin{array}{c}\text { Element } \\
\text { type }\end{array}$ & $\begin{array}{c}\text { Number of } \\
\text { nodes }\end{array}$ & $\begin{array}{c}\text { Number of } \\
\text { elements }\end{array}$ \\
\hline CAX $4 \mathrm{H}$ & 85541 & 36732 \\
\hline
\end{tabular}

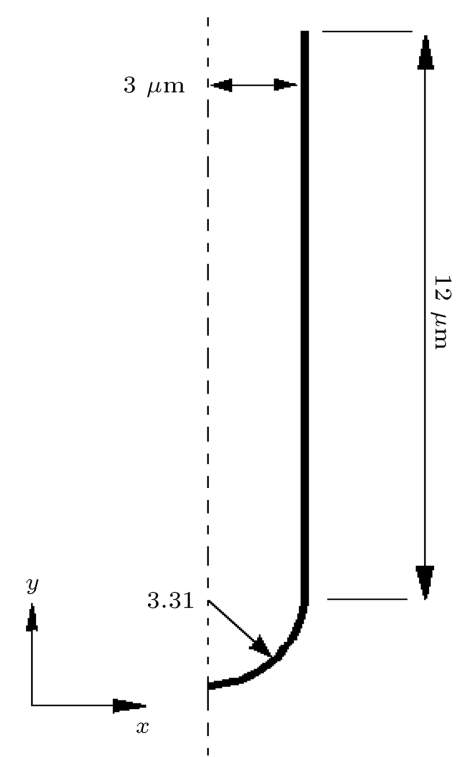

Figure 4. Indenter geometry and dimension in finite element model.

The interactions between the deformable slave cell body and the rigid indenter as well as those between the cell and the rigid substrate are considered as surfacebased contact pairs including the faces of the elements in the contact regions. The surface of the cell is moisturized; therefore, it has been assumed that there is no friction between indenter and cell in the contact points. Also, the adhesion between the surfaces has been ignored. Because of the large deformation of the cell, finite sliding has been allowed between the surfaces in contact regions. A static loading is applied to the indenter and the indenter is slowly displaced downwards from $0 \mathrm{um}$ to the depth of $22 \mathrm{um}$ for mouse oocyte and from $0 \mathrm{um}$ to the depth of $25 \mathrm{um}$ for mouse embryo, and the reaction forces are measured. Loading is done in 11 displacement steps, each of which requires 20-22 iterations. The models are analyzed in Abaqus/standard.

The procedure of inverse analysis for determination of hyperelastic parameters is as follows $[24,44,45]$ :

1. The finite element model of cell injection experiment is run by Abaqus software, which includes the initial hyperelastic parameters;

2. The output data of Abaqus are written in the 'Ith.dat' file;

3. The output data are read in the ' $i$-th.dat' file by Matlab software;
4. The new hyperelastic parameters are estimated by Matlab and input file of Abaqus is modified based on the new estimation;

5. The modified finite element model is run and outputs are transmitted to Matlab again;

6. The Jacobean is evaluated based on two different outputs, and the Hessian matrix is computed from Marquardet parameter, $\lambda$, and the Jacobean matrix;

7. The new hyperelastic parameters are estimated, Abaqus input file is modified, and finite element model is run again;

8. If the limited condition of fitness function, $\delta$, is satisfied, the iteration is finished and the optimized hyperelastic parameters could be obtained.

The defined fitness function form is as $[44,45]$ :

$$
\delta=\frac{1}{n} \sum_{i=1}^{n}\left[\frac{F_{\exp }(i)-F_{\operatorname{sim}}(i)}{F_{\exp }(i)}\right]^{2},
$$

in which $F_{\text {exp }}$ is the experimental force, $F_{\text {sim }}$ is the simulated force, and $n$ is the total number of the data.

In this study, Levenberg-Marquardt optimization method is used due to its high efficiency in process time in comparison with other techniques such as genetic algorithm or neural network. For example, finding optimized parameters for mouse oocyte cell using NeoHookean model needs 88 minutes (for 50 iterations), which means that each finite element simulation needs about 1.75 minutes to run while other techniques need more time.

\section{Result and discussions}

The hyperelastic material coefficients of mouse oocyte and embryo cells for different hyperelastic material models obtained by inverse finite element analysis are shown in Table 2.

Table 2 presents the hyperelastic parameters for both mouse oocyte and embryo cells. In this table, the obtained parameters by the Mooney-Rivlin model are same as those obtained by the polynomial form, while those obtained by Neo-Hookean are equal to those obtained by reduced polynomial forms. It is in the validation of the IFE model in our study that for material parameter, $N=1$ (see Eqs. (12) and (13)), the obtained values of these two sets of models are the same. The obtained hyperelastic parameters indicate that all models predict almost the same quantity for mouse oocyte and embryo cells. By comparing different hyperelastic material parameters of mouse oocyte and embryo cells by different models (see Table 2), it can be observed that, in all models, the material parameters of mouse embryo are greater than those of mouse oocyte. 
Table 2. Material parameters of mouse oocyte and embryo cells for different hyperelastic models resulting from inverse finite element model.

\begin{tabular}{|c|c|c|c|c|c|c|}
\hline \multirow{2}{*}{$\begin{array}{l}\text { Material model } \\
\text { Arruda-Boyce }\end{array}$} & \multicolumn{3}{|c|}{ Mouse oocyte } & \multicolumn{3}{|c|}{ Mouse embryo } \\
\hline & $\mu=0.0101$ & $D=0.0070$ & $\lambda_{m}=7$ & $\mu=0.0136$ & $D=0.0115$ & $\lambda_{m}=7$ \\
\hline Mooney-Rivlin & $C_{10}=0.0030$ & $C_{01}=0.0014$ & $D_{1}=0.0084$ & $C_{10}=0.0041$ & $C_{01}=0.0017$ & $D_{1}=0.0103$ \\
\hline Neo-Hookean & $C_{10}=0.0051$ & & $D_{1}=0.0106$ & $C_{10}=0.0069$ & & $D_{1}=0.0125$ \\
\hline Ogden & $\mu=0.0100$ & $\alpha=1.9700$ & $D=0.0011$ & $\mu_{1}=0.0131$ & $\alpha=1.9700$ & $D=0.0089$ \\
\hline Polynomial & $C_{10}=0.0030$ & $C_{01}=0.0014$ & $D_{1}=0.0084$ & $C_{10}=0.0041$ & $C_{01}=0.0017$ & $D_{1}=0.0103$ \\
\hline Reduced polynomial & $C_{10}=0.0051$ & & $D_{1}=0.0106$ & $C_{10}=0.0069$ & $D_{1}=0.0125$ & \\
\hline $\operatorname{Van}$ & $\mu=0.0089$ & $\alpha=0.0111$ & $D=0.0122$ & $\mu=0.0117$ & $\alpha=0.0135$ & $D=0.0144$ \\
\hline vall der vadis & $\beta=0.1099$ & & $\lambda_{m}=10$ & $\beta=0.1102$ & & $\lambda_{m}=10$ \\
\hline
\end{tabular}

Table 3. Evaluated Young's modulus, Poisson's ratio, initial bulk modulus, initial shear modulus, and objective function for mouse oocyte cell.

\begin{tabular}{lccccc}
\hline Material model & $\boldsymbol{E}(\mathbf{M P a})$ & $\boldsymbol{\nu}$ & $\boldsymbol{k}_{\mathbf{0}}(\mathrm{MPa})$ & $\boldsymbol{\mu}_{\mathbf{0}}(\mathrm{MPa})$ & $\boldsymbol{\delta}$ \\
\hline Arruda-Boyce & 0.0308 & 0.5 & 284.6643 & 0.0103 & 0.0049 \\
Mooney-Rivlin & 0.0264 & 0.5 & 237.2902 & 0.0088 & 0.0054 \\
Neo-Hookean & 0.0308 & 0.5 & 187.8095 & 0.0103 & 0.0047 \\
Ogden & 0.0300 & 0.5 & 1813.7 & 0.0100 & 0.0045 \\
Polynomial & 0.0264 & 0.5 & 237.2902 & 0.0088 & 0.0054 \\
Reduced polynomial & 0.0308 & 0.5 & 187.8095 & 0.0103 & 0.0047 \\
Van der Waals & 0.0267 & 0.5 & 164.4130 & 0.0089 & 0.0050 \\
\hline
\end{tabular}

Table 4. Evaluated Young's modulus, Poisson's ratio, initial bulk modulus, initial shear modulus, and objective function for mouse embryo cell.

\begin{tabular}{lccccc}
\hline Material model & $\boldsymbol{E}(\mathbf{M P a})$ & $\boldsymbol{\nu}$ & $\boldsymbol{k}_{\mathbf{0}}(\mathbf{M P a})$ & $\boldsymbol{\mu}_{\mathbf{0}}(\mathbf{M P a})$ & $\boldsymbol{\delta}$ \\
\hline Arruda-Boyce & 0.0414 & 0.5 & 173.7256 & 0.0138 & 0.4720 \\
Mooney-Rivlin & 0.0347 & 0.5 & 193.7921 & 0.0116 & 0.3737 \\
Neo-Hookean & 0.0416 & 0.5 & 159.7731 & 0.0139 & 0.4751 \\
Ogden & 0.0394 & 0.5 & 225.7533 & 0.0131 & 0.3983 \\
Polynomial & 0.0347 & 0.5 & 193.7921 & 0.0116 & 0.3737 \\
Reduced polynomial & 0.0416 & 0.5 & 159.7731 & 0.0139 & 0.4751 \\
Van der Waals & 0.0352 & 0.5 & 139.1338 & 0.0117 & 0.3793 \\
\hline
\end{tabular}

Results indicate that embryo cell is much stiffer than oocyte cell.

The values of elasticity module, $E$, Poisson's ratio, $\nu$, initial shear modulus, $\mu_{0}$, initial bulk modulus, $k_{0}$, and fitness function, $\delta$, of mouse oocyte and embryo cells are evaluated and presented in Tables 3 and 4 .

In Tables 3 and 4 , it can be seen that the calculated Poisson's ratio of mouse oocyte and embryo cells for all hyperelastic material models is 0.5 , which shows that the materials of mouse oocyte and embryo cells are compressible.

In addition, the elastic moduli of mouse oocyte obtained by hyperelastic material models are different; the predicted values of elastic moduli by ArrudaBoyce, Neo-Hookean, and reduced polynomial models are larger than those by other models (see Table 3 ) and those obtained by Neo-Hookean and reduced polynomial models are the largest (see Table 4). The reported elastic modulus of mouse embryo cell by Sun et al. [33] is $42.2 \mathrm{kPa}$. As can be seen, in some models, this value is very close to the values reported in Table 4, which are between $34.7 \mathrm{kPa}$ to $41.6 \mathrm{kPa}$; However, for mouse oocyte, the value reported by sun et al. is $17.9 \mathrm{kPa}$, while the values in Table 3 are between $26.4 \mathrm{kPa}$ to $30.8 \mathrm{kPa}$. This difference is basically because of two different datasets used by the two studies. Reported values by Sun et al. are the results of interpolation between 3 groups of datasets while this study, because of limitation, just uses one group of the mentioned data sets.

By taking the values of fitness function, $\delta$, into 
account, it could be seen that among these different hyperelastic materials, Ogden model is more suitable for characterizing mouse oocyte cell (see Table 3), while for mouse embryo cell, Mooney-Rivlin as well as polynomial form shows the best performance (with lower $\delta$ ) (see Table 4). It could also be concluded that for large strains, for mouse embryo and oocyte cells, Mooney-Rivlin, and Ogden material models are more suitable. These values of elastic modulus are significantly lower than those for zebrafish embryo's elastic moduli [46] and greater than those for red blood cell membrane $[46,47]$, indicating that, in comparison, the constitutive materials of mouse oocyte and embryo cells are much softer than zebrafish embryos and stiffer than red blood cell membrane.

It is clear that Ogden and Mooney-Rivlin models are best fitted for simulation of mouse oocyte and embryo cells. Normalized hyperelastic parameters of Ogden and Mooney-Rivlin models versus the number of iterations are shown in Figures 5 and 6.

The simulation indicates that after 7-8 iterations, the parameter values asymptotically approach their constant values.

The force-deformation results obtained from simulation of mouse oocyte and embryo cells using the IFEM in comparison with the experimental data have been presented in Figures 7 and 8 .

As can be seen in these figures, the simulated

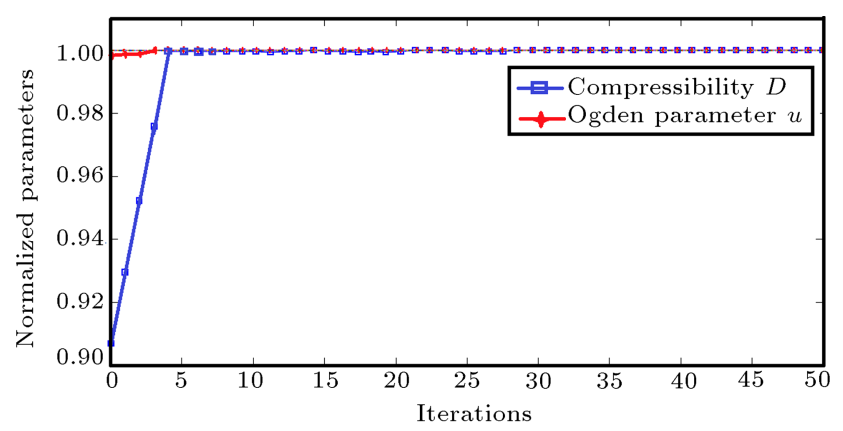

Figure 5. Normalized hyperelastic material parameters for mouse oocyte.

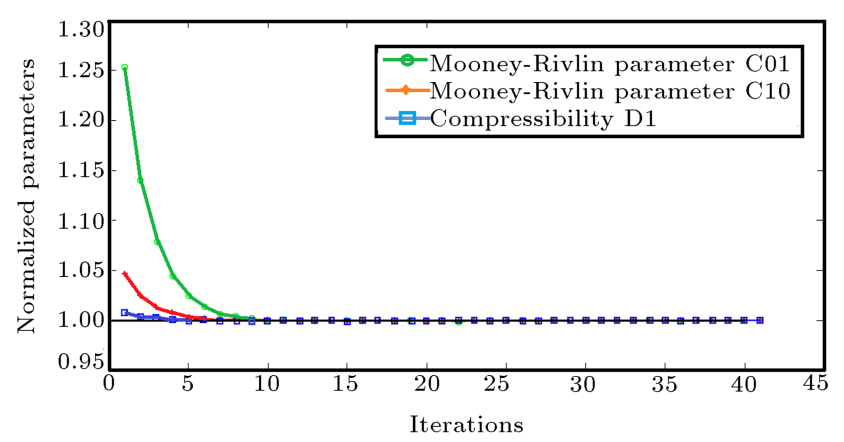

Figure 6. Normalized hyperelastic material parameters for mouse embryo.

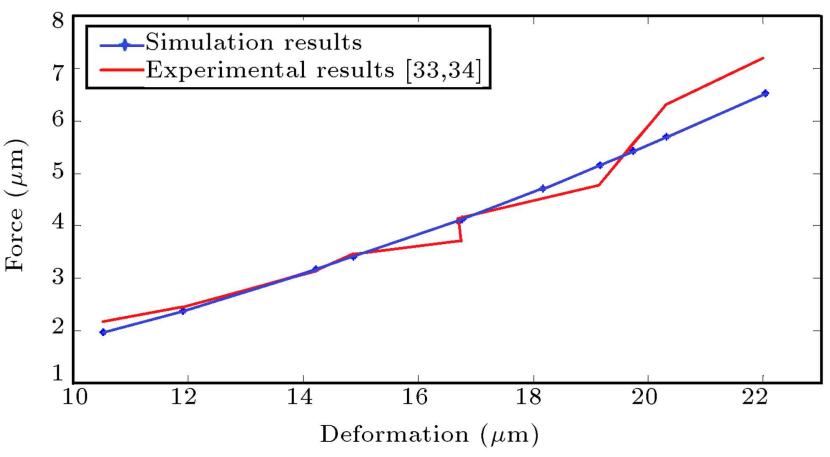

Figure 7. Simulated force-deformation in comparison with experimental data for mouse oocyte.

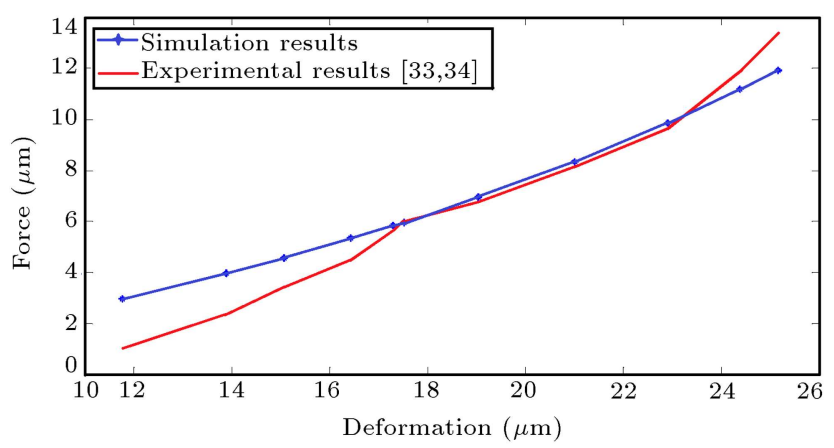

Figure 8. Simulated force-deformation in comparison with experimental data for mouse embryo.

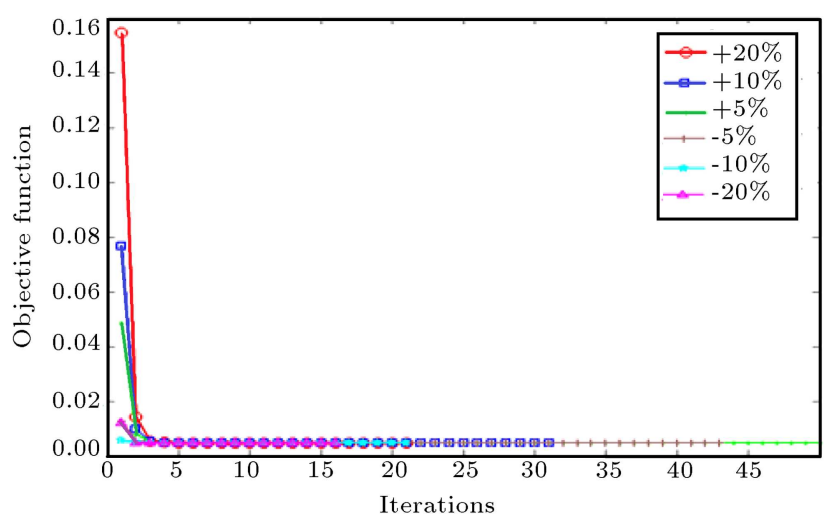

Figure 9. Sensitivity analysis results for mouse oocyte cell.

results are in good agreement with the experimental data.

Levenberg-Marquardt optimization algorithm is sensitive to the initial guess [48]. The sensitivity analysis on initial guesses within $\pm 5 \%, \pm 10 \%$, and $\pm 20 \%$ is presented in Figures 9 and 10 .

In Figures 9 and 10, different initial guesses converge on their final values, which shows that this algorithm is not sensitive to initial guess or, at least, in the range of the considered initial guesses, this is the case.

Because of the nature of such biological cells and their constructed materials, among different hypere- 


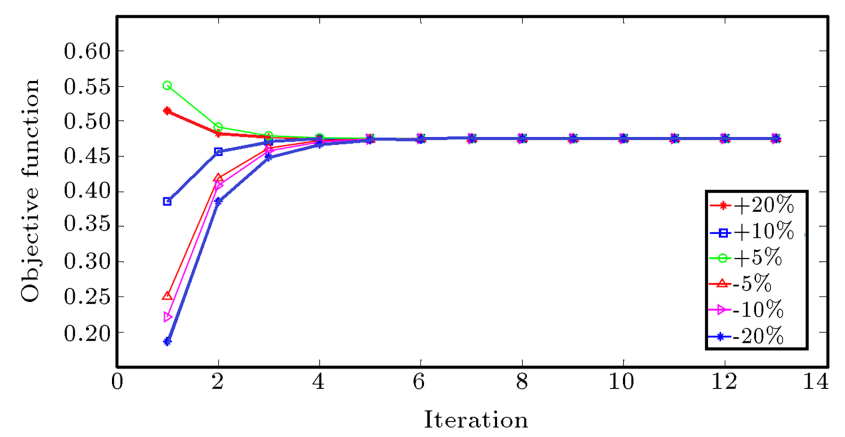

Figure 10. Sensitivity analysis results for mouse embryo cell.

lastic models, there are one or sometimes two models which can well describe the material of the cell; for example, Davies et al. [49] reported that Mooney-Rivlin model could better characterize behavior of the potato cell wall.

In addition, in the case of different growth stages of one cell type, it is possible that one model could describe one stage well while, for the next stage, the other model is better; for example, Tan et al. [46] reported that in Blastula stage of zebrafish embryo cell, the Mooney-Rivlin model could well describe it, while in other stages, such as segmentation and Pharyngula, Yeoh material, and Cheng material could well describe the cell, respectively .

As pointed out in Section 2, on material model, our study is based on the large deformation, but for further investigation and validation, the elasticity module of mouse embryo and oocyte cells has also been evaluated using the classical Hertz-Sneddon model, which is developed to be suitable for small deformations [50,51]. This classical model is based on two assumptions. The material of the cell is characterized using the linear elastic theory even in large deformations and, in order to be able to assume infinitesimal deformations, the size of the indenter tip should be small enough.

Three different tip geometries, namely, flat, conical, and parabolic, are used to investigate assumptions of Hertz-Sneddon model (Figure 11).

The evaluated Young moduli of mouse oocyte and
Table 5. Elasticity module for different tip geometries using Hertz-Sneddon model.

\begin{tabular}{lccc}
\hline Indenter & $\begin{array}{c}\text { Parabolic } \\
\text { tip }\end{array}$ & $\begin{array}{c}\text { Conical } \\
\text { tip }\end{array}$ & $\begin{array}{c}\text { Flat } \\
\text { tip }\end{array}$ \\
\hline Mouse embryo, $E(\mathrm{kPa})$ & 8.5944 & 21.5535 & 132.5335 \\
Mouse oocyte, $E(\mathrm{kPa})$ & 7.7898 & 15.3517 & 118.1663 \\
\hline
\end{tabular}

embryo cells using Hertz-Sneddon model are presented in Table 5 .

As could be seen in Tables 3 and 4, the evaluated Young modulus for mouse oocyte is $30 \mathrm{kPa}$ based on Ogden model, while for embryo cells, based on Mooney-Rivlin material model, it is $34.7 \mathrm{kPa}$; this values are different from those obtained by classical contact mechanics theory (Table 5). This indicates that classical contact mechanics is not valid for large deformations. Moreover, it shows that complex geometry between cell and indenter should also be considered in evaluation of elasticity module, which violates the second assumption in classical Hertz-Sneddon model.

Though the study uses hyperelastic models to extract the mechanical properties of biological cells, other models such as nonlinear fractional derivative model $[1,52,53]$ can also be adopted in the study; moreover, the research results can be explained in the layman way [54], which can be considered in future works for more comparison.

\section{Conclusion}

Biological cell studies have many applications in biology, cell manipulation, and diagnosis of the diseases such as cancers and malaria. A wide variety of experimental techniques and mechanical models have been developed to better analyze their mechanical behavior and internal physiological structure.

In some cases, it has been reported that more than 6 months of training are required for a biologist to get enough skill to manipulate and work with a cell [55]. Therefore, one application of such models could be in virtual reality based simulators to reduce the training time for a biologist.
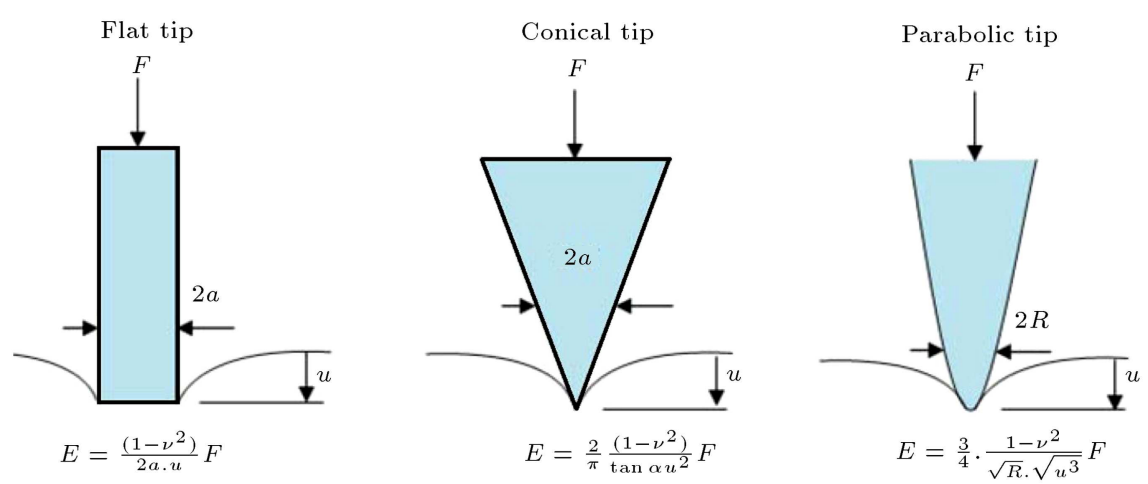

Figure 11. Indentation of cell by three different geometries of indenters: (a) Flat, (b) conical, and (c) parabolic. 
In this study, inverse finite element and Levenberg-Marquardt optimization algorithm were used to characterize constructive material of mouse oocyte and embryo cells in cell injection experiment. Different hyperelastic material models, namely, Arruda-Boyce, Mooney-Rivlin, Neo Hooke, Ogden, Polynomial, Reduced polynomial, and Van der Waals, were used to find the material model that could give the best fit between experimental and simulated forces. It was shown that Ogden material model could better characterize mouse oocyte cell, while Mooney-Rivlin material was more suitable for mouse embryo cell. Moreover, Poisson's ratio for all of the material models was 0.5 , which showed that constructive materials of both mouse oocyte and embryo cells were compressible. In this study, by taking the elastic moduli of mouse oocyte and embryo cells into account, it was seen that mouse oocyte cell was softer than mouse embryo cell by 30 percent. Also, the study showed that the classical contact mechanics models could not describe the phenomena caused by large deformations and complex geometry between the cell and indenter tip.

\section{Nomenclature}

$U \quad$ Strain energy potential function;

$C_{i j} \quad$ Temperature-dependent material parameter;

$\mu \quad$ Temperature-dependent material parameter;

$D \quad$ Incompressibility;

$\bar{I}_{1} \quad$ First deviatoric strain invariant;

$J^{e l} \quad$ Elastic volume ratio;

$\delta \quad$ Fitness parameter;

$F_{\exp } \quad$ Experimental force;

$F_{\text {sim }} \quad$ Simulated force;

$n \quad$ The number of data;

$P \quad$ Estimated hyperelastic parameter matrix;

$J \quad$ Jacobean matrix;

$H \quad$ Hessian matrix;

$\mu_{0} \quad$ Initial shear modulus;

$K_{0} \quad$ Initial bulk modulus;

$\nu \quad$ Poisson's ratio;

E Young's modulus.

\section{References}

1. Lim, C.T., Zhou, E.H., and Quek, S.T. "Mechanical models for living cells-a review", Journal of Biomechanics, 39, pp. 195-216 (2006).

2. Ladjal, H., Hanus, J.-L., and Ferreira, A. "Micro-tonano biomechanical modeling for assisted biological cell injection", IEEE Transactions on Biomedical Engineering, 60, pp. 2461-2471 (2013).

3. Tan, Y., Sun, D., Huang, W., and Cheng, S.H. "Mechanical modeling of biological cells in microinjection", IEEE Transactions on NanoBioscience, 7, pp. 257-266 (2008).

4. Dupire, J., Socol, M. and Viallat, A. "Full dynamics of a red blood cell in shear flow", Proceedings of the National Academy of Sciences, 109, pp. 20808-20813 (2012).

5. Chan, B., Li, C., Au-Yeung, K., Sze, K., and Ngan, A. "A microplate compression method for elastic modulus measurement of soft and viscoelastic collagen microspheres", Annals of Biomedical Engineering, 36, pp. 1254-1267 (2008).

6. Hochmuth, R.M. "Micropipette aspiration of living cells", Journal of Biomechanics, 33, pp. 15-22 (2000).

7. MacKay, J.L. and Kumar, S. "Measuring the elastic properties of living cells with atomic force microscopy indentation", Cell Imaging Techniques, pp. 313-329 (2013).

8. Zhong, M.-C., Wei, X.-B., Zhou, J.-H., Wang, Z.-Q., and Li, Y.-M. "Trapping red blood cells in living animals using optical tweezers", Nature Communications, 4, p. 1768 (2013).

9. Yarmush, M.L. and King, K.R. "Living-cell microarrays", Annual Review of Biomedical Engineering, 11, pp. 235-257 (2009).

10. Puig-De-Morales, M., Grabulosa, M., Alcaraz, J., Mullol, J., Maksym, G.N., Fredberg, J.J., and Navajas, D. "Measurement of cell microrheology by magnetic twisting cytometry with frequency domain demodulation", Journal of Applied Physiology, 91, pp. 1152-1159 (2001).

11. Laurent, V.M., Hénon, S., Planus, E., Fodil, R., Balland, M., Isabey, D., and Gallet, F. "Assessment of mechanical properties of adherent living cells by bead micromanipulation: Comparison of magnetic twisting cytometry vs optical tweezers", Journal of Biomechanical Engineering, 124, pp. 408-421 (2002).

12. Cañadas, P., Wendling-Mansuy, S. and Isabey, D. "Frequency response of a viscoelastic tensegrity model: Structural rearrangement contribution to cell dynamics", Journal of Biomechanical Engineering, 128, pp. 487-495 (2005).

13. Abbasi, A.A., Vossoughi, G., and Ahmadian, M. "Deformation prediction by a feed forward artificial neural network during mouse embryo micromanipulation", Animal Cells and Systems, 16, pp. 121-126 (2012).

14. Abbasi, A.A., Ahmadian, M., and Vossoughi, G. "Comparison of mouse embryo deformation modeling under needle injection using analytical Jacobian, nonlinear least square and artificial neural network techniques", Scientia Iranica, 18, pp. 1486-1491 (2011).

15. Abbasi, A.A., Sayyaadi, H., and Vossoughi, G. "Sensitivity analysis of mouse embryos in needle injection 
experiment using artificial neural network", 2nd International Conference on Future Information Technology (ICFIT 2011), Singapore, pp. 16-18 (2011).

16. Abbasi, A.A., Ahmadian, M., and Vossoughi, G. "Deformation prediction of mouse embryos in cell injection experiment by a feedforward artificial neural network", ASME 2011 International Design Engineering Technical Conferences and Computers and Information in Engineering Conference, American Society of Mechanical Engineers, Washington, DC, USA, pp. 543-550 (2011).

17. Ahmadian, M., Vossoughi, G., Abbasi, A., and Raeissi, P. "Cell deformation modeling under external force using artificial neural network", Journal of Solid Mechanics, 2, pp. 190-198 (2010).

18. Ahmadian, M., Vossoughi, G., Abbasi, A., and Raeissi, P. "Modeling of cell deformation under external force using neural network", ASME 2010 International Mechanical Engineering Congress and Exposition, American Society of Mechanical Engineers, 2, Vancouver, British Columbia, Canada, pp. 659-665 (2010).

19. Abbasi, A.A. and Ahmadian, M. "Prediction of reaction force on external indenter in cell injection experiment using support vector machine technique", $A S M E$ 2012 International Mechanical Engineering Congress and Exposition, American Society of Mechanical Engineers, 2, Houston, Texas, USA, pp. 537-543 (2012).

20. Abbasi, A.A., Barari, A., and Ahmadian, M. "Deformation prediction of biological cells in cell injection experiment using support vector machine modeling", Proceedings of the Canadian Society for Mechanical Engineering International Congress, Winnipeg, Manitoba, Canada (2012).

21. Abbasi, A.A., Vossoughi, G., and Ahmadian, M. "Application of adaptive neural fuzzy inference technique for biological cell modeling-part A: Deformation prediction", 2nd International Conference on Future Information Technology (ICFIT 2011), IPCSIT, Singapore, pp. 310-314 (2011).

22. Abbasi, A.A., Vossoughi, G., and Ahmadian, M. "Application of adaptive neural fuzzy inference technique for biological cell modeling-part B: Prediction of external applied force", 2nd International Conference on Future Information Technology (ICFIT 2011), IPCSIT, Singapore (2011).

23. Abbasi, A.A., Ahmadian, M., and Barari, A. "Biological cell modeling under needle injection experiment", Proceedings of the Canadian Society for Mechanical Engineering International Congress, Winnipeg, Manitoba, Canada (2012).

24. Abbasi, A.A. and Ahmadian, M.T. "Large deformation characterization of mouse oocyte cell under needle injection experiment", Amirkabir International Journal of Modeling, Identification, Simulation and Control, 44, pp. 21-25 (2012).

25. Namani, R. and Simha, N. "Inverse finite element analysis of indentation tests to determine hyperelastic parameters of soft-tissue layers", The Journal of Strain Analysis for Engineering Design, 44, pp. 347-362 (2009).

26. Delalleau, A., Josse, G., Lagarde, J., Zahouani, H., and Bergheau, J. "Characterization of the mechanical properties of skin by inverse analysis combined with the indentation test", Journal of Biomechanics, 39, pp. 1603-1610 (2006).

27. Slaboch, C.L., Alber, M.S., Rosen, E.D., and Ovaert, T.C. "Mechano-rheological properties of the murine thrombus determined via nanoindentation and finite element modeling", Journal of the Mechanical Behavior of Biomedical Materials, 10, pp. 75-86 (2012).

28. Liu, K., VanLandingham, M.R., and Ovaert, T.C. "Mechanical characterization of soft viscoelastic gels via indentation and optimization-based inverse finite element analysis", Journal of the Mechanical Behavior of Biomedical Materials, 2, pp. 355-363 (2009).

29. Needham, D. and Hochmuth, R. "A sensitive measure of surface stress in the resting neutrophil", Biophysical Journal, 61, pp. 1664-1670 (1992).

30. Tsai, M.A., Frank, R.S., and Waugh, R.E. "Passive mechanical behavior of human neutrophils: power-law fluid", Biophysical Journal, 65, pp. 2078-2088 (1993).

31. Hibbit, K. ABAQUS Theory and User Manuals Version 6.9, USA: ABAQUS Inc (2009).

32. "MATLAB 2008a, 1994-2010, The MathWorks," Inc., available at: www. mathworks. com.

33. Sun, Y., Wan, K.-T., Roberts, K.P., Bischof, J.C., and Nelson, B.J. "Mechanical property characterization of mouse zona pellucida", NanoBioscience, IEEE Transactions on, 2, pp. 279-286 (2003).

34. Flückiger, M. "Cell membrane mechanical modeling for microrobotic cell manipulation", Diploma Thesis, ETHZ Swiss Federal Institute of Technology, Zurich, WS03/04 (2004).

35. Arruda, E.M. and Boyce, M.C. "A three-dimensional constitutive model for the large stretch behavior of rubber elastic materials", Journal of the Mechanics and Physics of Solids, 41, pp. 389-412 (1993).

36. Samur, E., Sedef, M., Basdogan, C., Avtan, L., and Duzgun, O. "A robotic indenter for minimally invasive measurement and characterization of soft tissue response", Medical Image Analysis, 11, pp. 361-373 (2007).

37. Mooney, M. "A theory of large elastic deformation", Journal of applied physics, 11, pp. 582-592 (1940).

38. Rivlin, R., "Large elastic deformations of isotropic materials. IV. Further developments of the general theory", Philosophical Transactions of the Royal Society of London. Series A, Mathematical and Physical Sciences, 241, pp. 379-397 (1948).

39. Ogden, R.W., Non-linear Elastic Deformations, Dover Publications, pp. 532 (1997).

40. Macosko, C.W., Rheology: Principles, Measurement and Applications, VCH Publishers, p. 550 (1994). 
41. Ogden, R. "Large deformation isotropic elasticity-on the correlation of theory and experiment for incompressible rubberlike solids", Proceedings of the Royal Society of London. A. Mathematical and Physical Sciences, 326, pp. 565-584 (1972).

42. Rivlin, R.S., and Saunders, D. "Large elastic deformations of isotropic materials. VII. Experiments on the deformation of rubber", Philosophical Transactions of the Royal Society of London. Series A, Mathematical and Physical Sciences, 243, pp. 251-288 (1951).

43. Kilian, H.-G. "Equation of state of real networks", Polymer, 22, pp. 209-217 (1981).

44. Abbasi, A.A., and Ahmadian, M.T. "Whole cell mechanical property characterization based on mechanical properties of its cytoplasm and bio membrane in cell injection experiment using inverse finite element algorithm", Proceedings of the ASME 2012 International Mechanical Engineering Congress \& Exposition, 2, Houston, Texas, USA, (2012).

45. Abbasi, A.A., and Ahmadian, M. "Deformation characterization of mouse oocyte cell using inverse finite element and levenberg-Marquardt optimization algorithm in needle injection experiment", ASME 2012 International Design Engineering Technical Conferences and Computers and Information in Engineering Conference, American Society of Mechanical Engineers, Chicago, Illinois, USA, pp. 847-852 (2012).

46. Tan, Y., Sun, D., Huang, W., and Cheng, S.H. "Characterizing mechanical properties of biological cells by microinjection", IEEE Transactions on Nanobioscience, 9, pp. 171-180 (2010).

47. Hochmuth, R.M., Mohandas, N., and Blackshear, P.L. "Measurement of the elastic modulus for red cell membrane using a fluid mechanical technique", Biophysical Journal, 13, pp. 747-762 (1973).

48. Lei, F. and Szeri, A. "Inverse analysis of constitutive models: biological soft tissues", Journal of Biomechanics, 40, pp. 936-940 (2007).

49. Davies, G.C., Hiller, S., and Bruce, D.M. "A membrane model for elastic deflection of individual plant cell walls", Journal of Texture Studies, 29, pp. 645667 (1998).

50. Kim, Y., Shin, J.H., and Kim, J. "Atomic force microscopy probing for biomechanical characterization of living cells", Biomedical Robotics and Biomechatronics, 2008. BioRob 2008. 2nd IEEE RAS and EMBS International Conference on, IEEE, pp. 889894 (2008).

51. Cross, S.E., Jin, Y.-S., Rao, J., and Gimzewski, J.K. "Nanomechanical analysis of cells from cancer patients", Nature Nanotechnology, 2, pp. 780-783 (2007).

52. He, J.H. "A tutorial review on fractal spacetime and fractional calculus", Int. J. Theor. Phys., 53(11), pp. 3698-3718 (2014).

53. Wang, K. and Liu, S. "A new solution procedure for nonlinear fractional porous media equation based on a new fractional derivative", Nonlinear Science Letters A, 7, pp. 135-140 (2016).

54. Ayati, Z., Ansari Joubeni, F., and Ahmady, S. "Comparison of optimal homotopy asymptotic method and homotopy perturbation method for Telegraph equation", Nonlinear Science Letters A, 7, pp. 99-108 (2016).

55. Inoue, K., Tanikawa, T., and Arai, T. "Micromanipulation system with a two-fingered micro-hand and its potential application in bioscience", Journal of Biotechnology, 133, pp. 219-224 (2008).

\section{Biographies}

Ali Asghar Abbasi received a BS degree in Robotics Engineering from Shahrood University of Technology, Shahrood, Iran, in 2008. He received his MS degree in Mechatronics Engineering from the Department of Mechanical Engineering at Sharif University of Technology, Tehran, Iran, in 2011. His current research includes cell mechanics, micro/nano manipulation with mechanical devices, and intelligent systems.

Mohammd Taghi Ahmadian received a PhD degree from the Department of Mechanical Engineering at the University of Kansas, USA, in 1986. From 1984 to 1985, he was Assistant Professor at the University of Missouri, and from 1985 to 1988, he was Assistant Professor in the Department of Mechanical Engineering at the University of Kansas at Lawrence. He is currently Professor in the School of Mechanical Engineering at Sharif University of Technology, Tehran, Iran. His research interests include cellular biomechanics, soft tissue modeling, strength of material, and advance finite element method.

Ali Alizadeh received a $\mathrm{PhD}$ degree from Sharif University of Technology, Tehran, Iran, in 2013. He was also accepted as a post-doc in the Institute of Biotechnology and Environment (IBE) at Sharif University of Technology, in 2015. He is currently Assistant Professor in the field of Nanobiotechnology and his main research interests are developing novel drug delivery systems for small molecules as well as protein delivery by applying both experimental and theoretical methods.

Sina Tarighi received a BS degree in Industrial Engineering from Sharif University of Technology, Tehran, Iran, in 2008. He received his MA degree in MBA from the Graduate School of Management and Economy at Sharif University of Technology, Tehran, Iran, in 2012. He is now PhD student of Technology Management at Iran University of Science and Technology, beginning his studies in 2014. 